Communications in Physics, Vol. 24, No. 3 (2014), pp. 257-266

DOI:10.15625/0868-3166/24/3/3981

\title{
THE VATLY RADIO TELESCOPE: PERFORMANCE STUDY
}

\author{
NGUYEN THI PHUONG, PHAM NGOC DIEP, PIERRE DARRIULAT, PHAM TUYET \\ NHUNG, PHAM TUAN ANH, PHAM NGOC DONG, DO THI HOAI, NGUYEN THI THAO \\ VATLY, INST, 179, Hoang Quoc Viet, Cau Giay, Ha Noi, Vietnam.
}

E-mail: phuonghus91@gmail.com

Received 07 May 2014

Accepted for publication 30 June 2014

\begin{abstract}
A detailed account of the performance of the VATLY radio telescope, operated in Ha Noi on and near the $21 \mathrm{~cm} \mathrm{HI} \mathrm{line,} \mathrm{is} \mathrm{given.} \mathrm{Drift} \mathrm{scans} \mathrm{across} \mathrm{the} \mathrm{Sun} \mathrm{are} \mathrm{used} \mathrm{to} \mathrm{measure} \mathrm{the} \mathrm{dependence} \mathrm{of} \mathrm{the} \mathrm{gain} \mathrm{on} \mathrm{frequency} \mathrm{and}$ power, revealing small nonlinearities at or below the percent level. Interferences associated with the electromagnetic pollution in the Ha Noi environment are described. The sensitivity of the instrument is discussed and demonstrated with the detection of the Crab. The reliability of the measurement of the spectral flux density is illustrated by comparing solar flux data measured in Ha Noi and at the Australian observatory of Learmonth.
\end{abstract}

Keywords: Radio Telescope, $21 \mathrm{~cm}$ HI line, sensitivity.

\section{INTRODUCTION}

The VATLY radio telescope is operated in Ha Noi (Vietnam) at or near the frequency of the HI $21 \mathrm{~cm}$ line, $1.420 \mathrm{GHz}$. Its main features have been described in earlier publications [1] and observations of the disk of the Milky Way [2] and of the Sun [3] have been previously reported.

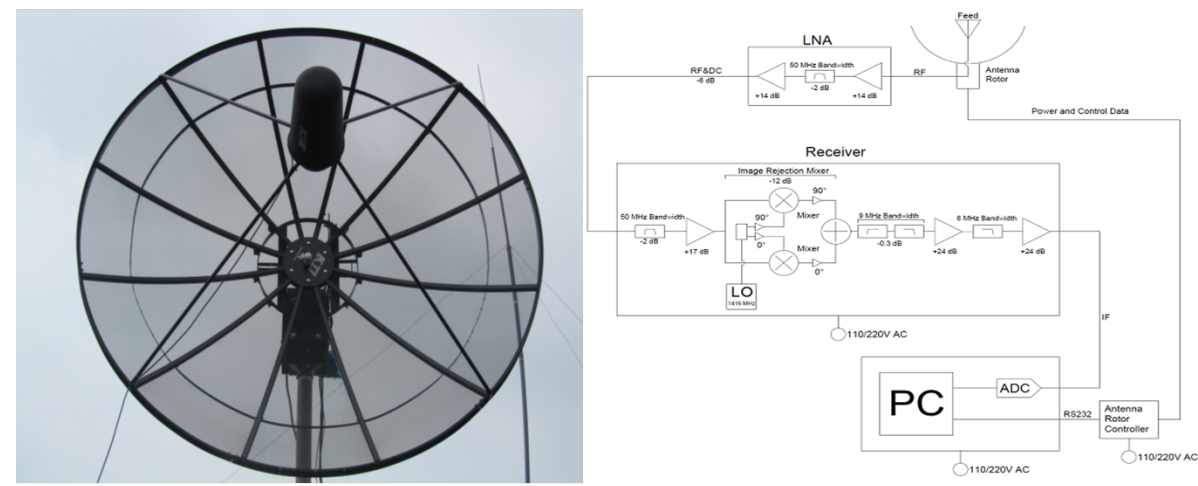

Fig. 1. Close-up view of the antenna and feed horn (left panel) and block diagram of the electronics [4] (right panel).

(C)2014 Vietnam Academy of Science and Technology 
The telescope is equipped with a fully steerable parabolic dish, $2.6 \mathrm{~m}$ in diameter, remotely adjustable in elevation and azimuth (Fig. 1). The reflected power is collected at the focus, where it is locally pre-amplified, shifted to lower frequency using standard super-heterodyne, amplified and digitized. Standard data collection consists in a sequence of successive measurements of $\sim 8$ $\mathrm{s}$ duration each, digitized in the form of a frequency histogram covering $\sim 1.2 \mathrm{MHz}$ in $156 \mathrm{bins}$ of $\sim 7.8 \mathrm{kHz}$ each. This is obtained by stitching together three adjacent bandwidths. Such a typical distribution is shown in Fig. 2 (right). The $21 \mathrm{~cm}$ hydrogen line is clearly seen above a slowly varying continuum. It signals the presence of hydrogen clouds in the field of view and is associated with electron spin flip in the hydrogen atom. The continuum signals the presence of ionized matter and is associated with thermal or "free-free" (bremsstrahlung) emission, including synchrotron radiation from free relativistic electrons accelerated in the interstellar magnetic field. Galaxies such as ours contain many HI clouds and the $21 \mathrm{~cm}$ signal of the disk of the Milky Way is particularly strong. On the contrary, the Sun emits exclusively in the continuum.

The telescope orientation is remotely adjustable and a small TV camera allows watching the antenna movement from the control room below where a desktop displays the data being recorded and other relevant information. The angular aperture of the main lobe (the "beam") is well described by a Gaussian having a $\sigma$ of $2.3^{\circ}$ and the pointing accuracy is measured to be $0.22^{\circ}$ in $a \times \cos (h)$ and $0.11^{\circ}$ in $h$ where $a$ and $h$ are the azimuth and elevation respectively [1].

The following sections describe the performance of the instrument with particular emphasis on the factors that limit its sensitivity, reliability and accuracy.

\section{DRIFT SCANS ACROSS THE SUN}

\section{II.1. General features}

Drift scans across the Sun are a convenient source of information allowing for following the antenna temperature from the normal sky level up to that of the Sun, both in the continuum and on the $21 \mathrm{~cm}$ line. The procedure is illustrated in Fig. 2. Data are collected over two hours starting at time $t(\mathrm{hr})$, the telescope pointing to the position at which the Sun passes at time $t+1$. This allows for the study of three regions, labelled 1 to 3 in the figure, corresponding to periods before, during and after Sun crossing. The frequency spectra, using a central frequency of $1420.4 \mathrm{MHz}$, allow for defining a continuum level by linear interpolation on either side of the $21 \mathrm{~cm} \mathrm{HI} \mathrm{line} \mathrm{and}$ for evaluating the contribution of the line by subtraction of the continuum. While the Sun does not emit significantly on the line, its continuum contribution, in quiet state, is typically a factor $\sim 6$ above that of the empty sky.

\section{II.2. Frequency dependence of the gain}

Over the bandwidth, the frequency dependence of the continuum is expected to be negligible. The decrease observed in Fig. 2, of nearly $10 \%$ in total, suggesting a HI absorption feature is in fact a purely instrumental artefact. A linear fit to the continuum of the form $a i+b$, where $i$ labels the frequency bin, allows for the study of the dependence of $a$ on $b$ when scanning across the Sun and on the central frequency $f$ by taking drift scans at different $f$ values. The result, displayed in Fig. 3, shows that $a$ is nearly proportional to $b$ and varies linearly with $f$.

The relative gain drop per frequency channel is $0.55 \%$ (i.e. $\sim 70 \mathrm{ppm} / \mathrm{kHz}$ ) at a central frequency of $1420.4 \mathrm{MHz}$ and decreases by $80 \mathrm{ppm}$ (i.e. $\sim 10 \mathrm{ppm} / \mathrm{kHz}$ ) per $\mathrm{MHz}$ of central frequency. 

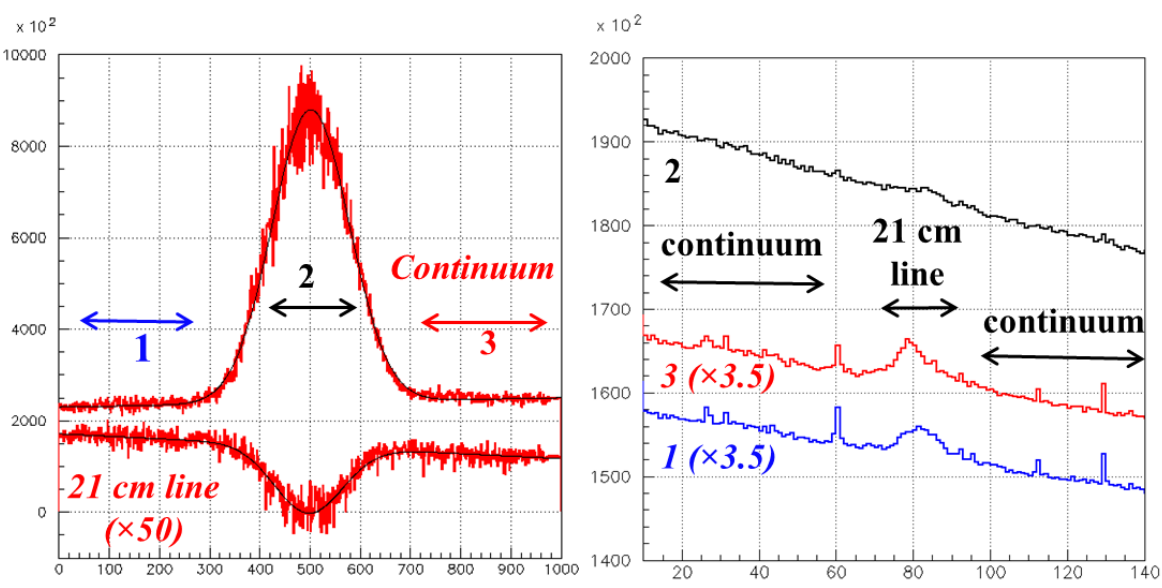

Fig. 2. Left panel: time dependence of the spectral flux density (arbitrary units) for the continuum and the $21 \mathrm{~cm}$ line (multiplied by 50) separately; the abscissa, in measurement numbers, covers two hours. Right panel: frequency spectra measured before (down, 100300) during (top, 450-650) and after (middle, 750-950) Sun crossing. The middle and lower spectra have been multiplied by 3.5 for convenience.
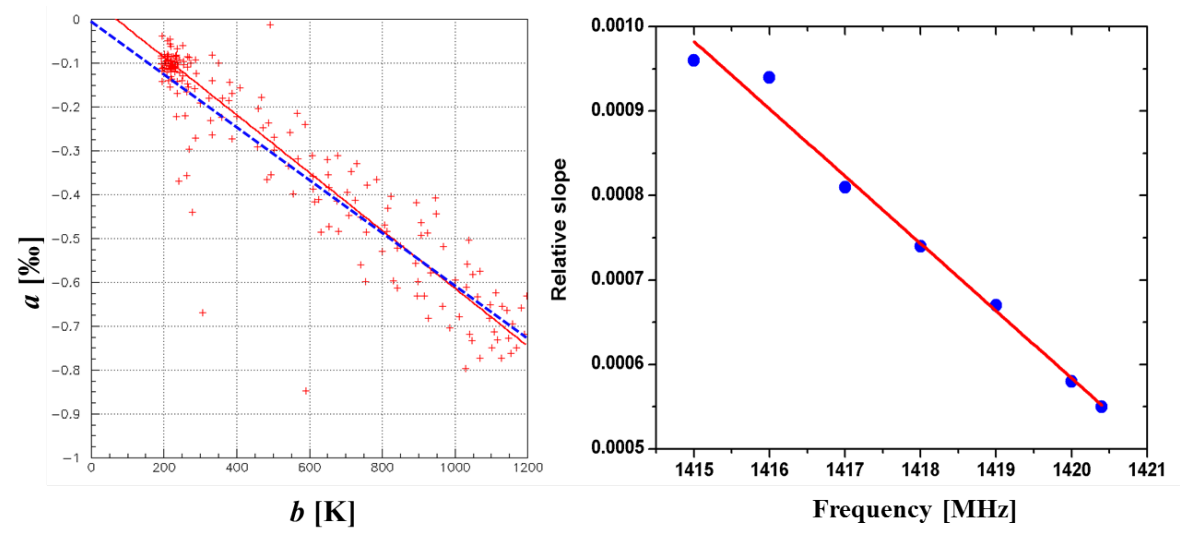

Fig. 3. Left panel: dependence of $a(\%)$ on $b(\mathrm{~K})$. The dotted line shows perfect proportionality as a reference. Right panel: dependence of $-a / b$ on central frequency $(\mathrm{MHz})$.

\section{II.3. Non-linearity of the response}

When scanning across the Sun, the contribution of the $21 \mathrm{~cm}$ line is seen to drop to zero (Figs. 2 and 4). The cause is instrumental as the Sun covers a negligible part, at the percent level, of the field of view: it results from a small non linearity of the response causing too large a continuum subtraction on the Sun. Indeed, the contribution of the Sun to the $21 \mathrm{~cm}$ line is obtained by subtracting off-the-Sun from on-the-Sun two large numbers: the total contribution (line+continuum). However, in both cases, the line is a minor fraction of the continuum and a small lack of linearity causes a large overestimate of the quantity to be subtracted. Assuming a 
non-linearity proportional to the signal, i.e. a quadratic response with a linear dependence of the gain on amplitude, it is sufficient to have a gain $4.6 \%$ times lower on the Sun than off the Sun to explain the effect, or, equivalently, $6.2 \%$ smaller on the Sun than at zero amplitude.

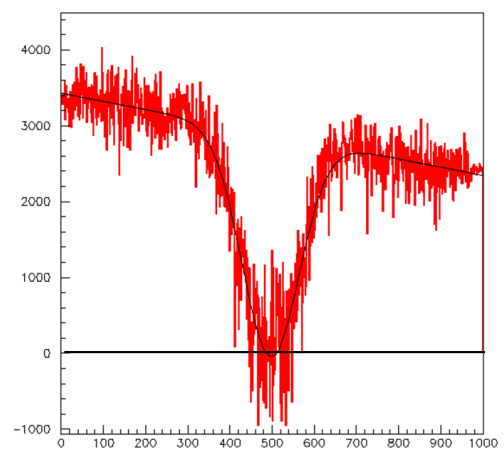

Fig. 4. The $21 \mathrm{~cm}$ line integrated between frequency channels 78 and 91 and over 74 drift scans of two hours each is displayed as a function of measurement number (500 corresponding to the Sun position).

\section{II.4. Small corrections related with the 3-bandwidth structure}

The three bandwidth structure of the frequency spectra is revealed by small imperfections of the response: slight differences, of the order of $2.5 \% \pm 1.5 \%$, between their respective gains and, for each of these, a slight enhancement in the middle of the bandwidth with respect to its edges as illustrated in Fig. 5. The latter is well described by a parabolic shape having a same sagitta in each of the three bandwidths. It has a broad distribution with a mean value of $1.8 \%$ and an rms value of $1.6 \%$.

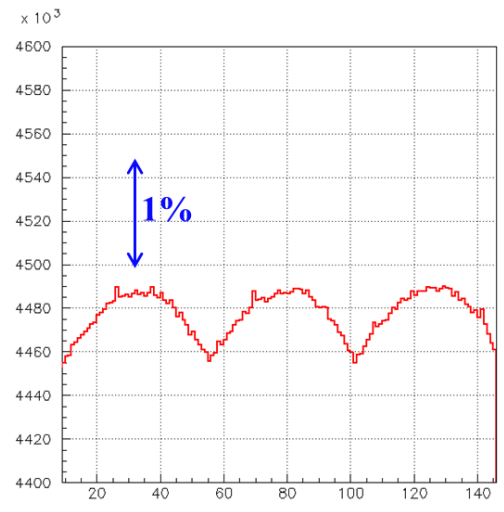

Fig. 5. Three-bandwidth structure of a frequency spectrum corrected for the frequency dependence of the gain discussed in Sec. II.2. Here, the relative sagitta of the parabolic bumps is $\sim 6 \%$, more than twice the average value. 


\section{INTERFERENCES}

Spikes, occurring in a few adjacent frequency bins, or bumps, covering some 20 adjacent bins, namely $\sim 150 \mathrm{kHz}$, are occasionally observed. By changing the value of the central frequency, one can verify that they occur at well defined frequencies. Both spikes and bumps have relative amplitudes with respect to the underlying continuum at the few percent level, eventually reaching a few $10 \%$.

\section{III.1. Bumps and spikes in the frequency spectrum}

Fig. 6 displays data collected at $1417.6 \mathrm{MHz}$ at $10^{\circ}$ elevation and $190^{\circ}$ azimuth during the night of 17 to 18 February 2014. The frequency spectrum shows a spike and a bump, the amplitudes of which are observed to switch on and off at well defined times, providing evidence for their human origin. Note that the spike remains present during the whole night ( $7 \mathrm{pm}$ to $7 \mathrm{am}$ ), however at much lower amplitude than during the day.
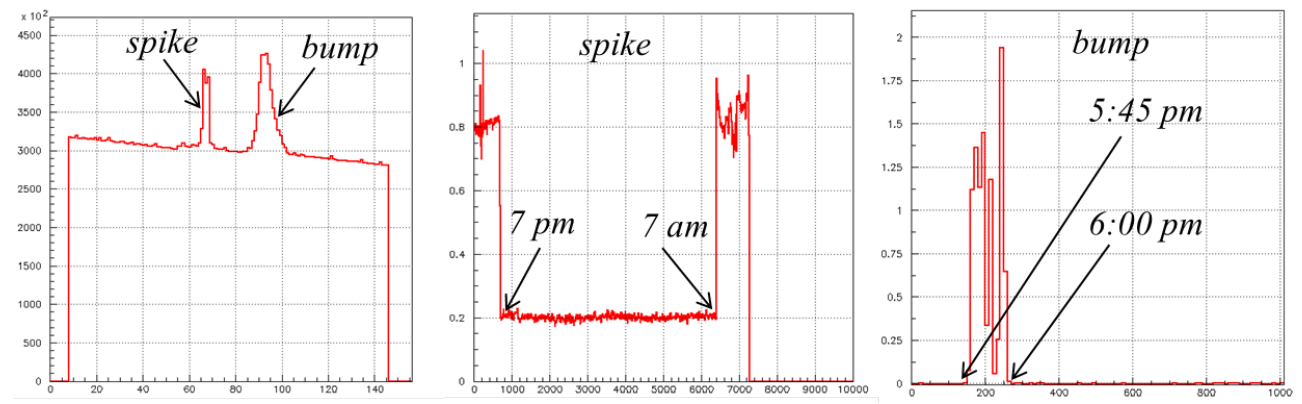

Fig. 6. Interferences of human origin. Frequency spectrum (left) and time dependences of the relative spike and bump amplitudes (centre and right).

Mapping the sky around this direction reveals the presence of another bump, also of human origin, at an azimuth of $180^{\circ}$. While the spike is relatively well localised over a region consistent with the size of the main antenna lobe, the bumps are nearly twice as broad. Such behaviour is typical. Moreover, varying the central frequency in steps of $0.25 \mathrm{MHz}$ between 1415 and 1425 $\mathrm{MHz}$ reveals the existence of a whole sequence of bumps spaced by $1.2 \mathrm{MHz}$ typically and having a width of some $150 \mathrm{kHz}$ and a variety of amplitudes at the level of a few percent. Harmonics, reflections on obstacles, detection into side lobes or pick-up by the electronics contribute to such interferences, making it difficult to identify precisely their sources. They are usually easy to remove when reducing the data and do not significantly deteriorate the quality of the observations.

However, the situation is more complex when the central frequency is tuned on the HI 21 $\mathrm{cm}$ line. In such cases, emission from the local ISM is known [5] to produce narrow lines at large galactic latitudes with line widths at the $\mathrm{km} / \mathrm{s}$ scale, namely at the scale of a frequency bin $(\sim 1.7$ $\mathrm{km} / \mathrm{s}$ ) in the present data. Similarly, at low galactic latitudes, HI clouds are known to produce broader bumps covering several $10 \mathrm{~km} / \mathrm{s}$, possibly significantly Doppler shifted, generating a broad enhancement extending over the full spectrum. It is therefore important to tell spikes and bumps having a $\mathrm{HI}$ origin from those having a human origin. A systematic survey of interferences in this frequency range has been made by mapping the sky over a broad range of azimuths and elevations $\left(120^{\circ}\right.$ to $240^{\circ}$ in steps of $30^{\circ}$ and $15^{\circ}$ to $75^{\circ}$ in steps of $20^{\circ}$ respectively) at a central frequency of 

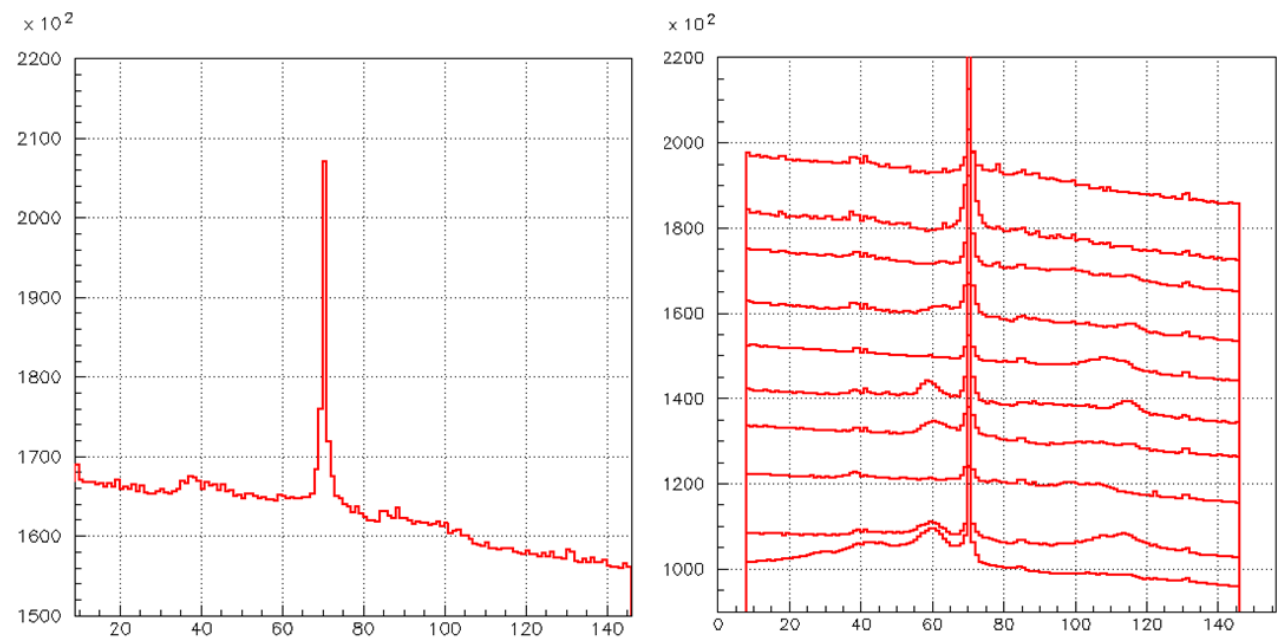

Fig. 7. Left: Summed frequency spectrum measured at $1420.3 \mathrm{MHz}$ on a 20 point grid covering the southern sky. Right: the same spectra separated in intervals of galactic latitude corresponding to bins of 0.1 units of its cosine; spectra have been normalised to be ordered from the galactic poles (up) to the galactic equator (Milky Way, down) for convenience.

1420.3 MHz and at 2 hour intervals in a sequence of ten scans covering a full afternoon and the following night. A number of bumps have been detected, at the few percent level, as well as a spike at a frequency of $\sim 1420.3 \mathrm{MHz}$ that is present everywhere. It illustrates the potential difficulty to tell the HI contribution, apparent at low latitude, from human interferences. In practical cases, however, it is easily overcome [2]. A beam switching technique may reduce the contamination by the galactic HI background. At low galactic latitudes the broad HI lines arise from the emission of cloud complexes in spiral arms lying along the line of sight. They can strongly affect the spectrum baseline.

\section{III.2. Spikes in the time distributions}

In addition to spikes occurring in the frequency spectrum there are also spikes occurring in the time distribution. As an illustration, Fig. 8 displays the time dependence of the antenna temperature in the evening of February 12th, 2014. The telescope was tuned on a central frequency of $1417.6 \mathrm{MHz}$ and pointed to $a=230^{\circ}, h=40^{\circ}$. A number of spikes are seen to occur, usually at the level of a few percent. The frequency spectra of the two largest of these spikes reveal major disturbances of the frequency slope and important gain differences between the three bandwidths. The cause may be a spike in the power line or in the ground or a transient being picked up by the electronics. We noted that in the case of large flares, when saturation occurs, similar dysfunctions take place. Here again, such spikes are easy to remove at data reduction level and do not deteriorate the quality of the observations. However, spikes display a continuous distribution toward low amplitudes, where they are not associated with any anomaly of the frequency spectrum and become progressively more frequent, particularly during daytime. Man-made interferences are particularly troublesome when one observes narrow interstellar lines at high galactic latitude, 
since it is ubiquitous and also affects the side lobes, depending on how the antenna is illuminated. They ultimately are the main limiting factor for the sensitivity of the instrument.
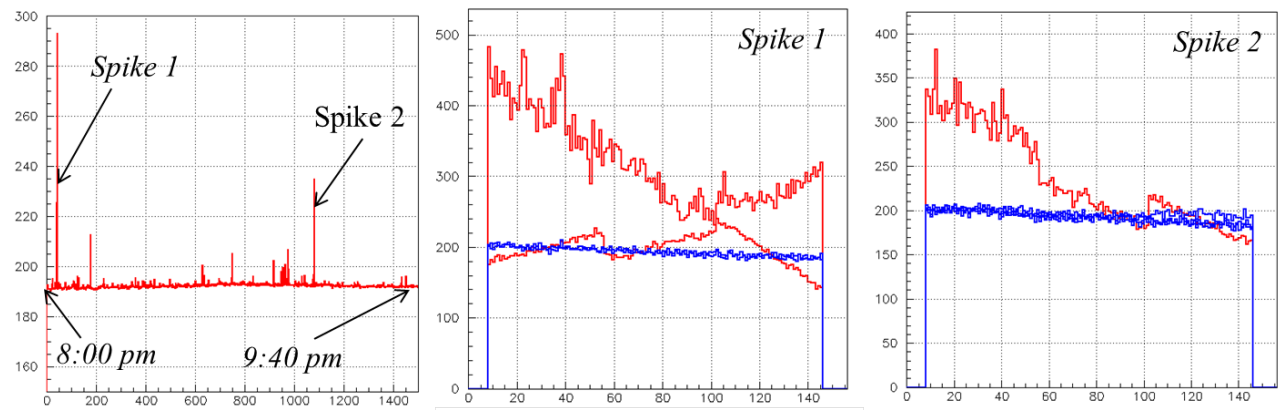

Fig. 8. Spikes in the time dependence of the antenna temperature. Left: a typical time distribution; centre and right: frequency spectra associated with the largest spikes. The spectra bracketing the spike are not affected.

\section{SENSITIVITY AND STABILITY}

In addition to external interferences, spikes and bumps, or the presence of multipath oscillations such as described in Reference 2, noise, including electronic noise as well as gain fluctuations due to other sources, limits the sensitivity of the instrument.

A first evaluation of the noise is obtained from the rms fluctuations of the antenna temperature around its mean when observing the Sun. Fig. 9 displays the distribution of the $\chi^{2}$ to a fit allowing for multipath oscillations covering the months of November and December 2013, using a reference uncertainty of $3 \%$. The mean value of the distribution, 1.58 , implies a mean noise level of $3 \sqrt{1.58}=3.8 \%$ of the solar signal, namely $\sim 5.3 \mathrm{~K}$.

A second evaluation is obtained from the spectral flux density recorded during quiet hours of the night between 12th and 13th February 2014. The data collected between 0:20 and 2:30 local time are split in 10 lumps of 100 measurements each, corresponding to $13.6 \mathrm{~min}$ for each lump. The flux in each lump is fit to a second order polynomial dependence on time with respect to which the rms deviation is calculated. For an average temperature of $191.5 \mathrm{~K}$, the mean rms fluctuation is measured to be $0.28 \mathrm{~K}$, namely a noise to signal ratio of $1.5 \%$.

Depending on where it occurs in the amplification chain, the noise can be expected to include a constant term and a term proportional to the signal. From the above examples, we may retain as a conservative estimate a background to noise ratio making it possible to detect signals at the permil level above background by making long enough observations.

A confirmation has been obtained by observing radio sources such as Taurus A (the Crab), expected at the $\sim 900 \mathrm{Jy}$ level. The latter illustrates well the practical limit of what can be reliably achieved on such sources. Fig. 10 left shows the detection of Taurus $A$ at the $\sim 1 \mathrm{~K}$ level over $\mathrm{a} \sim 250 \mathrm{~K}$ background from a set of $34 \mathrm{drift}$ scans across Taurus A. Also shown is the result of 21 drift scans across points that are shifted by $\pm 10^{\circ}$ of galactic longitude with respect to Taurus A. In such a case, the sensitivity is limited by the need to subtract spikes associated with human interferences, which could not be done reliably for significantly lower signals. 

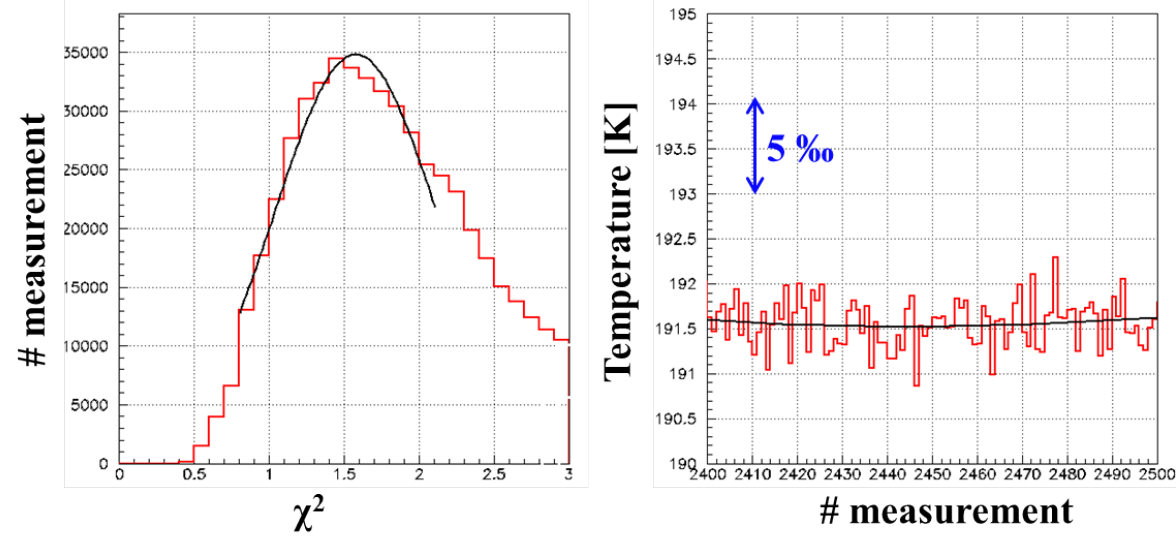

Fig. 9. Left panel: Distribution of the $\chi^{2}$ per degree of freedom, using arbitrary uncertainties of $3 \%$, to a fit of solar data allowing for multipath oscillations. Right panel: distribution of the temperature recorded during a February 2014 night in one of the ten 13.6 min lumps used for the noise analysis. The line shows the polynomial fit.
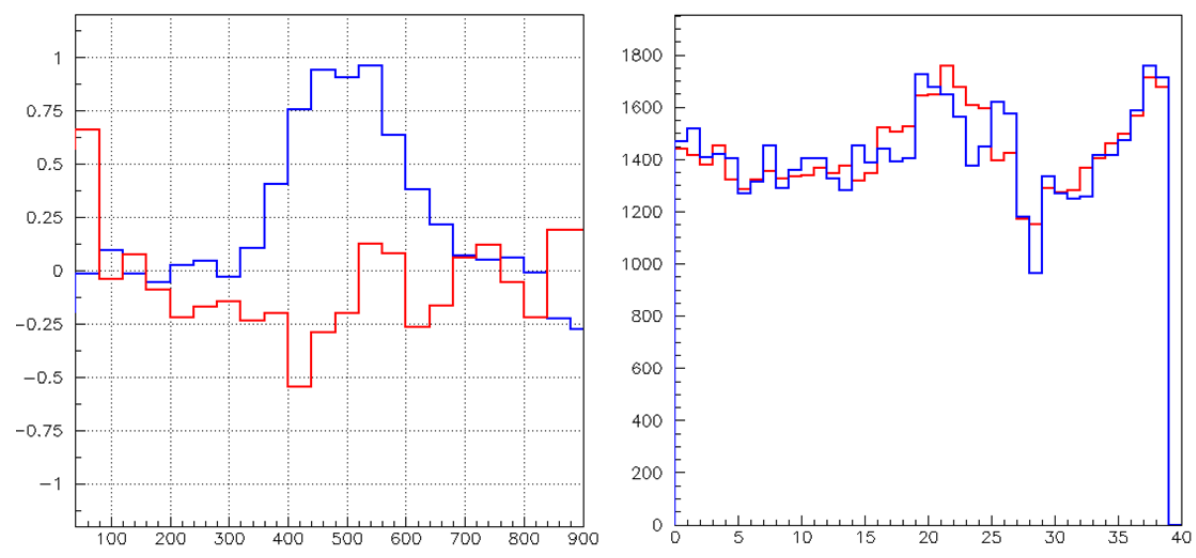

Fig. 10. Left: Antenna temperature $(\mathrm{K})$ averaged over 34 drift scans across Taurus A (the Crab, upper curve) and over 21 drift scans shifted by $\pm 10^{\circ}$ in galactic longitude. Right: Distribution of daily averaged solar fluxes measured in Learmonth (normalised to the $\mathrm{Ha}$ Noi system temperature in K) and Ha Noi from October 25th to December 9th 2013.

The daily averaged solar fluxes measured between October 25th and December 9th 2013 are compared in Fig. 10 with measurements made at the same frequency during the same period by the Learmonth solar observatory located at opposite latitude but same longitude as Ha Noi. The daily averaged rms deviations are $2.7 \%$ and $1.3 \%$ respectively, mostly due to slow drifts such as caused by changes in the ambient temperature. More precisely, the Hanoi data are of the antenna temperature, $1438 \mathrm{~K}$ on average, and the Learmonth data of the spectral flux density, $115 \mathrm{SFU}$ on average $\left(1 \mathrm{SFU}=10^{4} \mathrm{Jy}\right)$. Their ratio has a narrow distribution having a mean value of $12.5 \mathrm{~K} / \mathrm{SFU}$ with an rms deviation of $0.9 \mathrm{~K} / \mathrm{SFU}(7 \%)$ with respect to the mean. It corresponds to an antenna 
effective area of $3.45 \mathrm{~m}^{2}$ for a true area of $5.3 \mathrm{~m}^{2}$, namely an efficiency factor of $65 \%$. In Ha Noi, a calibration was performed each morning using a calibrated noise resistor and pointing to a fixed quiet region of the sky, measuring $206 \mathrm{~K}$ on average with an rms deviation with respect to the mean of $9 \mathrm{~K}(4.4 \%)$.

In summary, reliable measurements can be performed with an accuracy of a few permil over short periods and of a few percent over a whole day. Main limitations are uncontrolled slow gain drifts in the case of long observation times and small spikes caused by human interferences in the case of drift scans. In practice, it is difficult to reach sensitivities better than $\sim 1 \mathrm{~K}$, corresponding to $\sim 800 \mathrm{Jy}$. Rapidly switching on- and off-source should make it possible to ultimately reach sensitivities at the few 100 Jy level.

\section{SUMMARY AND CONCLUSIONS}

The performance of the VATLY radio telescope, operated in Ha Noi on and near the 21 $\mathrm{cm} \mathrm{HI}$ line, has been studied. Quantities such as the pointing accuracy $\left(0.11^{\circ} \times 0.22^{\circ}\right)$, the beam width $\left(\sigma=2.3^{\circ}\right)$ and the frequency resolution $(7.8 \mathrm{kHz})$ have been evaluated.

Drift scans across the Sun have revealed a small dependence of the gain on frequency, measured as a relative gain drop of $\sim 70 \mathrm{ppm} / \mathrm{kHz}$ at a central frequency of $1420.4 \mathrm{MHz}$, itself decreasing by $\sim 10 \mathrm{ppm} / \mathrm{kHz}$ per $\mathrm{MHz}$ of central frequency, implying a gain drop of nearly 130 $\mathrm{ppm} / \mathrm{kHz}$ at a central frequency of $1415 \mathrm{MHz}$. The gain was also observed to decrease when the amplitude of the detected signal increases, being 6.2\% smaller on the Sun than at zero amplitude, meaning a relative gain drop of $\sim 5 \mathrm{ppm} / \mathrm{K}$ of antenna temperature. A small modulation of the gain, in the form of three adjacent enhancements having sagittas at the level of a few permil, results from the stitching together of three separate bandwidths.

Human interferences have been found to be ubiquitous, either in the form of spikes in the frequency spectra, usually at well defined frequencies and sky coverage, or in the form of brief spikes affecting all frequencies for a single, or at most a few successive measurements. Such interferences practically limit the sensitivity of the instrument at the level of a few hundred Jy. Observations over extended periods suffer small gain drifts that imply daily averaged rms deviations of $\sim 2.7 \%$, typically twice as large as achieved at the Learmonth Australian solar observatory using a similar instrument. Comparison between simultaneous observations performed in Ha Noi and in Learmonth are consistent with an antenna efficiency factor of $\sim 65 \%$ and a conversion factor of $1.25 \mathrm{~K} / \mathrm{kJy}$ fluctuating with an rms deviation of $0.09 \mathrm{~K} / \mathrm{kJy}(7 \%)$ with respect to the mean. Very large spikes may occasionally occur, as do solar flares, causing major disturbance to the system.

The performance of the VATLY radio telescope as a training tool is remarkable and offers excellent opportunities for students to become familiar with the techniques and methods of radio astronomy. While giving access to detailed studies of strong radio sources, such as the Sun in the continuum or the disk of the Milky Way on the $21 \mathrm{~cm}$ line, its ability to detect sources of lesser strength is limited to a very few, such as Cygnus A (1600 Jy), Taurus A (900 Jy) or Cassiopeia A (2400 Jy).

The HI line is in principle an excellent tool to evaluate the distance of a galactic radio source by Doppler measurement of the absorption in a HI cloud in the foreground. However, the continuum antenna temperature of the background radio source should be well above the kinetic temperature (a few $10 \mathrm{~K}$ ) of the absorbing $\mathrm{HI}$ interstellar cloud and a larger antenna would be needed in this case. 


\section{ACKNOWLEDGMENT}

We thank Professor Nguyen Quang Rieu for encouragements and for very useful comments on the results presented here and on future possible observations. We express our gratitude to Dr Alain Maestrini and Dr Alan Rogers for their interest. We acknowledge financial support from the Vietnam National Foundation for Science and Technology Development (NAFOSTED) under grant number 103.08-2012.34, the Institute for Nuclear Science and Technology, the World Laboratory, the Odon Vallet Foundation and the Rencontres du Vietnam.

\section{REFERENCES}

[1] N.V. Hiep et al., The VATLY radio telescope, Comm. Phys.Vietnam, Vol 22, No 4 (2012), 365.

N.V. Hiep, Observation of the $21 \mathrm{~cm}$ sky using the VATLY radio telescope, Master thesis, presented at Vietnam Institute of Physics, 2012.

[2] N.V. Hiep et al., Mapping atomic hydrogen in the disk of the Milky Way, Comm. Phys. Vietnam, Vol 23, No 2 (2013), 107-119.

[3] N.V. Hiep et al., Radio Observation of Solar-Activity-Related mHz Oscillations, Sol. Phys. 289, 3 (2014), 939-950. P.N. Diep et al., Publications of the Astronomical Society of Australia 31(2014) e029 (7 pages); DOI:10.1017/pasa.2014.24

[4] A. Rogers, http://www.haystack.mit.edu/edu/undergrad/srt/oldsrt.html.

[5] H. Weaver and D.R.W. Williams, The Berkeley Low Latitude Survey of Neutral Hydrogen, Astron. \& Astrophys. Suppl., 17 (1974) 1-249. 